\title{
Avaliação nictemeral do perfil vertical da temperatura e do oxigênio da coluna d'água em viveiros de piscicultura escavado e de barragem
}

\section{Nictemeral evaluation of the vertical profile of temperature and dissolved of the water column in dugout and dam fish farming ponds}

\author{
Marcos Fernandes Silva' \\ Erlei Cassiano Keppeler²(*)
}

\section{Resumo}

O objetivo do presente estudo foi fazer uma avaliação nictemeral (24:00h), comparando um viveiro escavado e um viveiro de barragem, analisando a temperatura, saturação de oxigênio e oxigênio dissolvido (OD) no perfil da coluna d'água. Para tanto, foram realizadas leituras com oxímetro digital, nas profundidades de $0 ; 15 ; 30 ; 45 ; 60 ; 75 ; 90$ e $105 \mathrm{~cm}$. As leituras foram iniciadas às 09:00h, e foram feitas a cada 03:00h até que se completassem 24:00h. Houve diferença significativa em relação à saturação de oxigênio e ao OD. Quanto à temperatura, a maior amplitude térmica foi registrada, no viveiro escavado as 15:00h, encontrando-se uma termóclina na profundidade de 0 a $30 \mathrm{~cm}$. Em relação ao OD, o menor valor registrado na coluna d'água foi no viveiro escavado, na profundidade de $90 \mathrm{~cm}$, as 0:00h, chegando ao nível de 1,4 mg.L $\mathrm{L}^{-1}$ (quase considerado estado de anóxia). Constatamos que as variações nictemerais (24h.) na coluna d'água são mais abruptas e maiores nos viveiros escavados. Desta forma, o presente trabalho nos permitiu concluir que devido à maior renovação de água, os viveiros de barragem podem ser, socialmente e economicamente mais favoráveis para um piscicultor de baixa renda, pois favorece melhores condições de cultivo.

Palavras Chaves: variação nictemeral, temperatura, oxigênio.

\section{Abstract}

The aim of this study was carry out nictemeral evaluation (24:00h), comparing a dugout pond and dam pond, analyzing temperature, oxygen saturation, and dissolved oxygen (OD) in the profile of the water column. Therefore, we performed readings with digital oximeter at depths of $0,15,30,45,60,75,90$ and $105 \mathrm{~cm}$. The readings began 09:00h, and were made every 03:00h. until 24:00h. were finished. There was a significant difference from oxygen saturation and OD. As for temperature, the higher temperature range was recorded in the nursery dug the 15:00h, finding a thermocline

1 Gr.; Engenharia Agronômica; Universidade Federal do Acre, UFAC, Campus de Cruzeiro do Sul, UFAC, Brasil; Mestrando em Ciências Ambientais, Bolsista CAPES, Discente da Licenciatura em Ciências Biológicas. Endereço: Universidade Federal do Acre - Universidade da Floresta, Centro Multidisciplinar do Campus Floresta. Canela Fina, Km 05, Gleba Formoso; CEP: 69980-000 - Cruzeiro do Sul, AC - Brasil.

2 Dr.; Aquicultura; Universidade Estadual Paulista Júlio de Mesquita Filho, UNESP, Brasil; Professora de Magistério Superior da Universidade Federal do Acre, Campus de Cruzeiro do Sul; Endereço: Universidade Federal do Acre Universidade da Floresta, Centro Multidiscipinar do Campus Floresta. Canela Fina, Km 05, Gleba Formoso; CEP: 69980000 - Cruzeiro do Sul, AC - Brasil. E-mail: erleikeppeler@gmail.com

\begin{tabular}{llllll}
\hline Ambiência & Guarapuava (PR) v.l5 n.I & p. $194-206$ & Jan/Abr 2019 & ISSN I808 - 025I
\end{tabular}


depth 0-30 $\mathrm{cm}$. Regarding the OD, the lowest value recorded in the water column was excavated in the nursery at a depth of $90 \mathrm{~cm}$, the $0: 00 \mathrm{~h}$, reaching the level of $1.4 \mathrm{mg}$. $\mathrm{L}^{-1}$ (almost considered state of anoxia). We found that the diel variations (24h.) in the water column were more abrupt and higher in excavated ponds. In this way, the present work allowed us to conclude that due to the greater water renewal the excavated ponds can be, socially and economically more favorable for a low-income fish farmer, because it favors better cultivation conditions.

Key-Words: nictemeral variation, temperature, oxygen.

\section{Introdução}

Variação nictemeral em tanques é um tema relevante, mas ainda pouco discutido, sendo importante citar Barreto et al. (2010) e Brito et al (2017), principalmente no que tange aos problemas de variação diária de temperatura e queda nos níveis de oxigênio dissolvido, os quais são fatores que podem causar a morte tanto de peixes como da comunidade planctônica (OSTRENSKY; BOEGER, 1998), pois a taxa de produção diária de oxigênio pode não ser suficiente para suportar a respiração de todos os organismos do tanque, especialmente à noite (CYRINO et al., 1987), causando prejuízos para os piscicultores.

É facilmente perceptível pelos piscicultores que existem bolsões de água com temperaturas diferentes nos tanques de piscicultura durante o período de cultivo. Segundo Esteves (1998), pelo fato de a água quente ser menos densa que a água fria, ela permanece na superfície e não se mistura, enquanto que a água fria sendo mais densa fica embaixo. Esse fenômeno é conhecido como estratificação térmica e, dependendo do local de cultivo, pode trazer uma série de consequências desfavoráveis à produção, como problemas com oxigênio dissolvido (OSTRENSKY;BOEGER, 1998). A fotossíntese é sempre maior onde incide maior quantidade de luz, ou seja, na superfície e, assim, a água do fundo não se mistura ao oxigênio produzido na superfície e o oxigênio não chega até o fundo (FERNANDES et al., 2004).

Existe uma correlação entre a estratificação do oxigênio, da temperatura com a abundância de plâncton, podendo acontecer até mesmo em tanques rasos. Há uma grande variação de oxigênio dissolvido entre o período do dia e da noite, sendo essas concentrações diretamente ligadas à proliferação de plâncton e, normalmente, os problemas de falta de oxigênio ocorrem no período da noite quando a produção de oxigênio dissolvido não é suficiente para suportar a respiração de todos os organismos aquáticos nesses horários (CYRINO et al., 1987).

Segundo Pádua (2001) e Diniz et al. (2002), as alterações na temperatura e do oxigênio dissolvido na água são influenciadas por componentes biológicos e químicos, além de fatores bióticos e abióticos, sendo esses componentes alterados ciclicamente num período de 24 horas (variação nictemeral).

Para Vinatea Arana (2004), o equilíbrio dinâmico entre os fatores químicos, físicos, biológicos e tecnológicos do empreendimento é responsável pelo sucesso do cultivo em uma propriedade. As informações sobre esses fatores são essenciais no processo produtivo.

De acordo com Barbosa e Padisák (2002), a coluna d'água, em um ambiente aquático, pode ser dividida em três estratos, o Epilímnio ou Superfície: que consiste na camada superior menos densa; Metalímnio ou Meio: é a camada intermediária, com grandes variações térmicas, onde se localiza a termóclina, região com descontinuidade térmica e que constitui uma barreira física entre os estratos superiores e inferiores; Hipolímnio ou Fundo: correspondente ao estrato inferior com águas mais densas, frias e relativamente calmas. 
Vários estudos relataram a variação nictemeral de temperatura e oxigênio dissolvido, no Brasil, como os realizados por Fisher et al. (1983) e Tundisi e Matsumura-Tundisi (1994). Fisher et al. (1983) observaram o Lago Calado na Amazônia, por um período de 24 horas, em 1980. Este ambiente apresentava uma profundidade de 9 metros e, abaixo dos 4 metros, o Lago teve condições anóxicas. O reservatório Barra Bonita-SP esteve sujeito a um permanente mixing, quando observaram o ambiente (TUNDISI; MATSUMURA-TUNDISI, 1994). O Lago Dom Helvécio-MG esteve sujeito a um período de extensa estratificação e alta estabilidade na coluna da água, e o esforço do vento no lago foi muito baixo devido às características morfométricas do lago e sua bacia (TUNDISI; MATSUMURA-TUNDISI, 1994).

Desta forma, o objetivo geral do presente trabalho foi analisar a variação nictemeral (24 horas) do perfil vertical da temperatura, da saturação e concentração de oxigênio dissolvido da coluna d'água de um tanque escavado e de um viveiro de barragem, utilizados na piscicultura. Hipotetizamos observar uma maior homogeneidade na coluna d'água no viveiro de barragem, por apresentar constante renovação da água. Por outro lado, no viveiro escavado, esperamos encontrar termóclina e estratificações do oxigênio dissolvido, bem como condição de anoxia no fundo da coluna d'água.

\section{Material e Métodos}

\section{ÁREA DE ESTUDO}

O presente trabalho foi conduzido no município de Cruzeiro do Sul, Acre, situado no extremo Oeste do Estado (latitude 070 37'52" Sul e longitude 72 40’12” Oeste). A área experimental apresentava 11 viveiros escavados, e 3 viveiros de barragem. O local foi escolhido devido ser uma propriedade de pequeno piscicultor, de fácil acesso, e que representa bem a maioria dos piscicultores do Acre. Portanto, foram sorteados aleatoriamente um viveiro escavado e um (1) viveiro de barragem ou açude, e determinado um ponto de coleta em cada viveiro (Figura 1), para acompanhamento dos perfis verticais de temperatura e do oxigênio dissolvido. 


\section{Figura 1 - Localização da área de estudo}

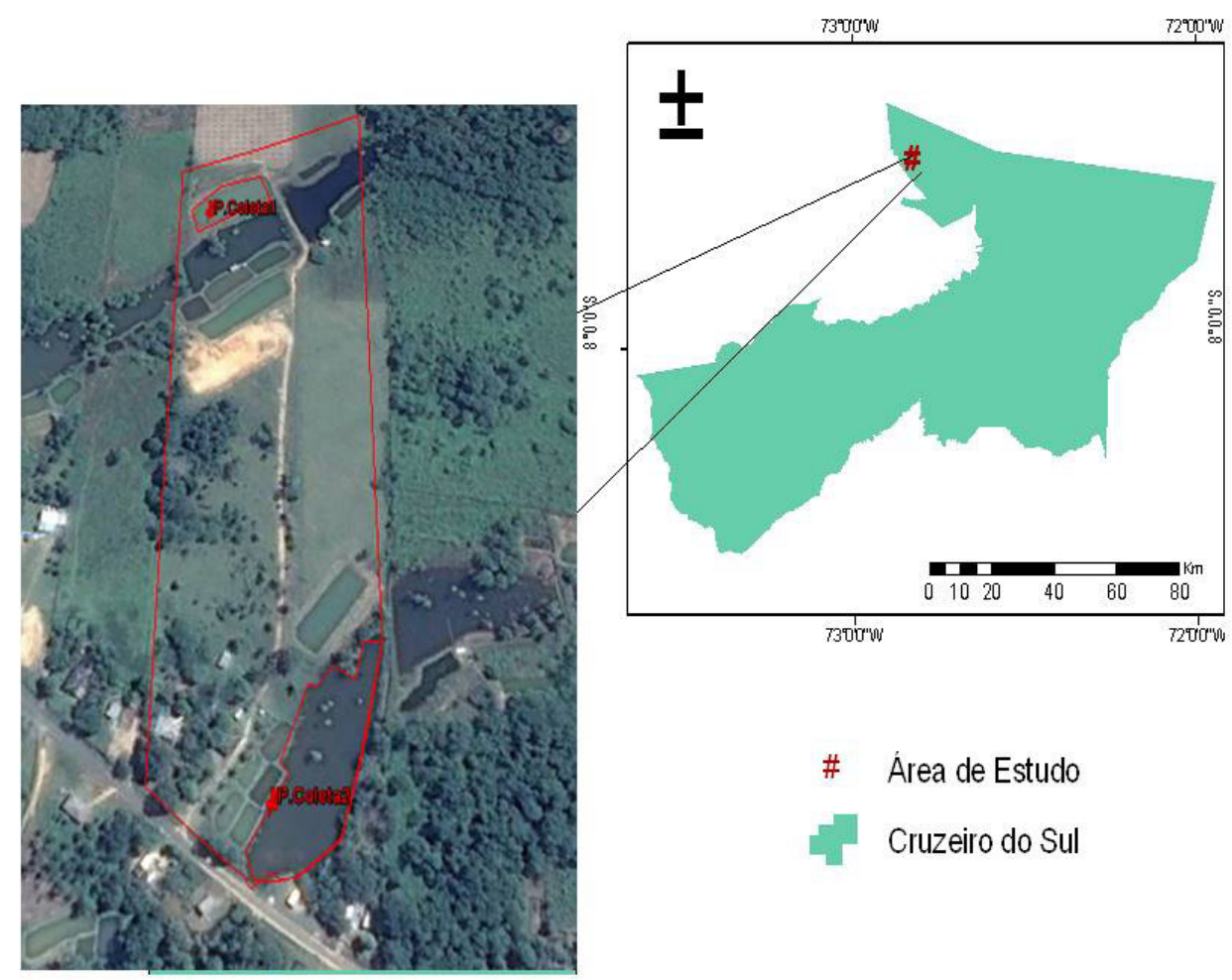

Segundo a classificação de Koppen, o clima de Cruzeiro do Sul pode ser caracterizado como quente e úmido e com sazonalidade bem definida (período chuvoso e seco), fenômeno esse que se dá devido a Zona de Convergência Intertropical na região (KELLER FILHO et al., 2005).

Em relação à vegetação do Acre, esta possui diversas formas e tipos, como vegetação florestal densa e com dossel relativamente homogêneo e fechado, e com árvores emergentes. Há uma predominância de palmeiras (Arecaceae) no dossel dessa floresta, especialmente nas áreas onde se concentra uma grande quantidade de igarapés formados por água preta, destacando a presença de Patauá - Oenocarpus bataua, Bacaba - Oenocarpus sp., Buriti - Mauritia flexuosa e Açaí - Euterpe oleraceae. Adicionalmente, é possível se observar uma vegetação florestal com dossel irregular e pouco denso e com poucas árvores emergentes, outra vegetação de Cruzeiro do Sul se apresenta com um dossel baixo, abundância de árvores finas e ausência de emergentes, outras áreas, com uma fisionomia aberta e predominantemente arbustiva, com árvores grandes escassas, cuja cobertura pouco densa, expõe o substrato formado por areia branca (SILVEIRA, 2003).

A formação do solo de Cruzeiro do Sul ocorre sobreposta a feições tipo terraço, oriundos de uma criação recente a partir da Formação Solimões, sendo sua maior exposição localizada a sudoeste da cidade de Cruzeiro do Sul, na confluência dos Rios Moa e Juruá. Ocorre ainda, uma deposição de sedimentos fluviais, flúvio-lacustre e em leques aluviais, que tem, em sua composição, coloração amarelo-escura, rosada e esbranquiçada, com intercalações de argilitos lenticulares, arenitos finos, friáveis, maciços, argilosos (CAVALCANTE, 2005). 
Segundo Cavalcante (2006), o solo de Cruzeiro do Sul também é constituído por sedimentos mais arenosos e, nos aluviões da planície fluvial, nas áreas de várzeas, estão as formações geológicas mais recentes que ocorrem ao longo dos rios e são compostas, predominantemente, de sedimentos inconsolidados (seixos, areia, argila).

As coletas foram nictemerais (24 horas), visando observar variações ao longo do dia e da noite na coluna d'água. $O$ viveiro escavado sorteado possuía uma área total de aproximadamente $2.250 \mathrm{~m}^{2}$, profundidade máxima de $0,90 \mathrm{~m}$ e mínima de $0,20 \mathrm{~m}$ no período de amostragem, podendo chegar à profundidade de até $1,5 \mathrm{~m}$ em períodos chuvosos, sem vegetação nas bordas e sem renovação de água. Foi construído há, pelo menos sete anos e, nos três últimos anos, é onde atualmente estava sendo cultivado Piau - Leporinus sp. Os peixes eram alimentados com ração durante todo o cultivo, caracterizando um cultivo semi-intensivo e a água renovada constantemente nos períodos de chuva.

O viveiro de barragem ou açude que foi construído há cerca de 40 anos, possuía uma área total de aproximadamente $12.300 \mathrm{~m}^{2}$. Nessa área, predominam pelo menos três tipos de vegetação em seu entorno: Junco - (Juncus effusus) da família Juncaceae, caracterizada como planta aquática; Capim (Quicuio - Brachiaria humidicola), da família Poaceae, caracterizado como pastagem; Planta Mosaico - (Ludwigia sedioides) da Família: Onagraceae, planta aquática. O viveiro de barragem com renovação de água durante todo o ano, era povoado com várias espécies de peixes entre elas, Piau - Leporinus sp., Curimatã - Prochilodus escrofa, Matrinxã - Brycon sp., Pirapitinga - Piaractus brachypomus. Estes peixes, geralmente, são alimentados com pouca ração e com a própria fauna aquática do açude, além de alimentos vegetais como caroço de cupuaçu, mandioca e jambo, caracterizando um cultivo extensivo.

As medidas dos parâmetros analisados foram realizadas com um medidor portátil digital (Ysi - Pro20), que fornece a medida de temperatura, saturação de oxigênio e oxigênio dissolvido. O sensor do equipamento foi colocado na água a cada profundidade determinada, até que os valores estabilizassem, passando para a profundidade seguinte. As leituras foram realizadas na superfície $(0,0 \mathrm{~m})$ e aos 0,$15 ; 0,30 ; 0,45 ; 0,60 ; 0,75 ; 0,90$ e $1,05 \mathrm{~m}$, no viveiro de barragem e na superfície $(0,0 \mathrm{~m})$ e aos 0,$15 ; 0,30 ; 0,45 ; 0,60 ; 0,75$ e $0,90 \mathrm{~m}$, no viveiro escavado, em intervalos de 3 horas a partir das 09 h do dia 18/10/2014 e finalizada às 09 h do dia 19/10/2014, até que se completasse 24 horas de coleta. Foram elaborados gráficos comparativos entre o viveiro escavado e o viveiro de barragem, correlacionando os horários, as leituras e as profundidades amostrais, para os parâmetros temperatura e oxigênio dissolvido.

Para a análise estatística, foi usado o T-Student a 5\% de significância com variâncias desiguais ou heterogêneas (BORGES; FERREIRA, 1999), para verificar se ocorreram diferenças significativas entre os dois tipos de viveiros pesquisados, e as variáveis amostradas, temperatura, saturação de oxigênio e oxigênio dissolvido, e os dados foram comparados como amostras independentes. O pacote utilizado foi o software Statistica (Statsoft, 2005),

\section{RESULTADOS E DISCUSSÃO}

Nos dois tipos de viveiros estudados, houve aumento da temperatura no período da tarde, sendo que a maior temperatura $\left(38,5^{\circ} \mathrm{C}\right)$ foi encontrada no viveiro escavado no horário de $15 \mathrm{~h}$, e, nesse horário, ocorreu a maior variação térmica na coluna d'água a temperatura caiu de $38,5^{\circ} \mathrm{C}$, na superfície para $28,4^{\circ} \mathrm{C}$ no fundo. 


\section{GRÁFICO 1 - Variação diária (Padrão nictemeral) do perfil vertical da Temperatura.}

Temperatura - $\mathrm{C}^{\circ}$

26283032343638

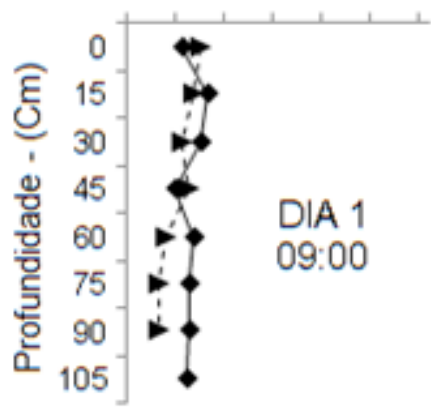

Temperatura - $\mathrm{C}^{\circ}$

$26 \quad 283032343638$

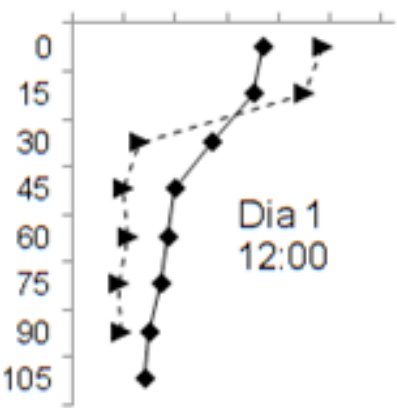

Temperatura - $\mathrm{C}^{\circ}$

$26 \quad 283032343638$

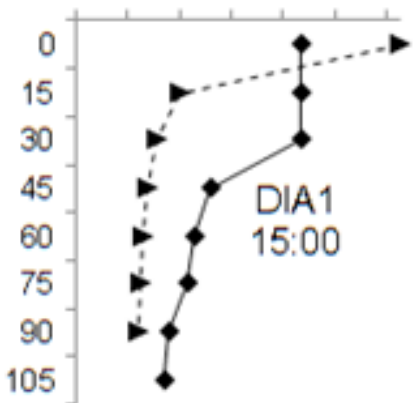

$\begin{array}{lllllll}26 & 28 & 30 & 32 & 34 & 36 & 38\end{array}$

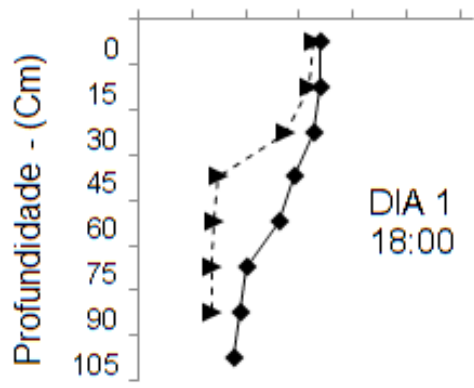

$\begin{array}{lllllll}26 & 28 & 30 & 32 & 34 & 36 & 38\end{array}$

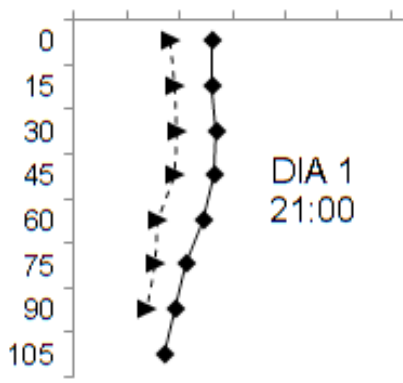

$\begin{array}{lllllll}26 & 28 & 30 & 32 & 34 & 36 & 38\end{array}$

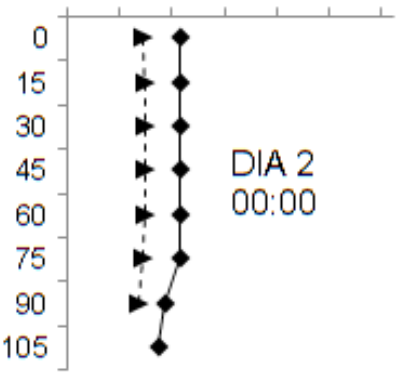

$\begin{array}{lllllll}26 & 28 & 30 & 32 & 34 & 36 & 38\end{array}$

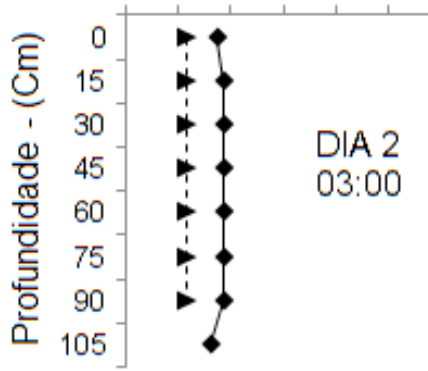

$\begin{array}{lllllll}26 & 28 & 30 & 32 & 34 & 36 & 38\end{array}$

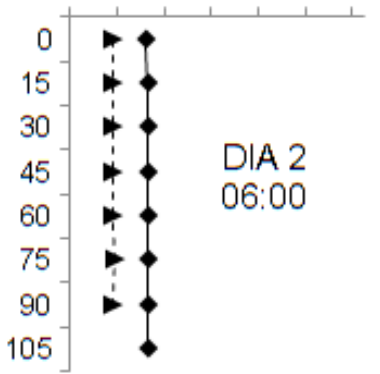

$\begin{array}{lllllll}26 & 28 & 30 & 32 & 34 & 36 & 38\end{array}$

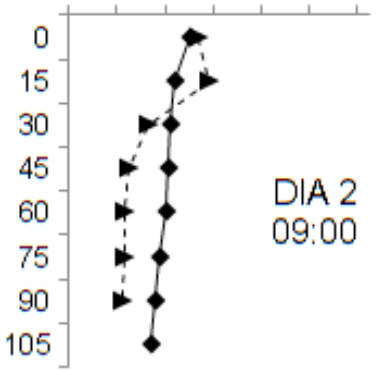

${ }^{-\cdots}=$ Viveiro Escavado; $\rightarrow-=$ Viveiro de Barragem.

A maior temperatura registrada no viveiro de barragem ou açude foi de $34,7^{\circ} \mathrm{C}$, também às $15 \mathrm{~h}$, e a menor temperatura encontrada neste ambiente foi de $28,5^{\circ} \mathrm{C}$, no fundo, às $09 \mathrm{~h}$. HenrySilva et al. (2010), em um trabalho realizado na Amazônia com camarões em viveiros escavados, encontrou, nesse mesmo horário (15h), uma amplitude térmica que variou de $33,5^{\circ} \mathrm{C}$, na superfície e chegou a $27,8^{\circ} \mathrm{C}$, no fundo, tendo uma variação de $5,7^{\circ} \mathrm{C}$ entre a superfície e o fundo. 
A partir das $21 \mathrm{~h}$, a temperatura na coluna d'água começou a se estabilizar, sendo que às 03h ela foi constante em todo o perfil da coluna d'água, voltando a ter variações a partir das 09h. Observou-se, também, que a estratificação permaneceu entre $12 \mathrm{~h}$ e $18 \mathrm{~h}$, do primeiro dia, não havendo mais estratificação até às 06h do segundo dia (Gráfico 1). Esteves et al. (1988), ao estudarem uma lagoa costeira tropical que tinha profundidade média de 1 metro (Lagoa Iodada, Rio de Janeiro) observaram estratificação térmica acentuada da coluna d'água durante o período iluminado do dia e desestratificação no período noturno.

No viveiro escavado, a temperatura, nos horários noturnos, permaneceu inferior à do viveiro de barragem, pelo menos $1,5^{\circ} \mathrm{C}$ (Gráfico 1 ). Em média, a temperatura, na coluna d'água, no viveiro escavado foi de $29,21^{\circ} \mathrm{C}$ e no viveiro de barragem de $30,24^{\circ} \mathrm{C}$. Segundo Kubitza (1999), a temperatura ideal para o cultivo de peixes tropicais varia de $28^{\circ} \mathrm{C}$ a $32^{\circ} \mathrm{C}$, logo os dois tipos de viveiros apresentaram condições favoráveis ao desenvolvimento da piscicultura.

Nos horários de $12 \mathrm{~h}$ e $15 \mathrm{~h}$, houve variação abrupta da temperatura no viveiro escavado entre as profundidades de 0 e $30 \mathrm{~cm}$, constatando-se a presença da termóclina (Gráfico 1). Já no viveiro de barragem essa variação foi menor e ocorreu entre a profundidade de $15 \mathrm{e} 45 \mathrm{~cm}$, às $12 \mathrm{~h}$ e, entre 30 e $45 \mathrm{~cm}$, às $15 \mathrm{~h}$.

Os níveis de saturação de oxigênio permaneceram superiores, no açude, em quase todos os horários, alcançando a maior amplitude entre os viveiros às $18 \mathrm{~h}$, com uma diferença de $54 \%$, na profundidade de $45 \mathrm{~cm}$ (Gráfico 2). Sendo que o valor mais alto registrado foi de $96 \%$ às $15 \mathrm{~h}$, no viveiro escavado, na profundidade de $0 \mathrm{~cm}$. Também, nesse mesmo viveiro e, no mesmo horário, houve a maior variação na coluna d'água com $96 \%$ na superfície $(0 \mathrm{~cm})$, chegando a $28 \%$ no fundo $(90 \mathrm{~cm})$. Henry-Silva et al. (2010), em um trabalho realizado em viveiros encavados com criação de tilápias, encontrou o maior valor de saturação (185\%), às $18 \mathrm{~h}$, sendo este valor encontrado logo abaixo da superfície e o mesmo relacionou este fato com a migração do fitoplâncton para regiões da coluna d'água que tem menor incidência de raios solares.

\section{GRÁFICO 2 - Variação diária (Padrão nictemeral) do perfil vertical da Saturação de Oxigênio.}
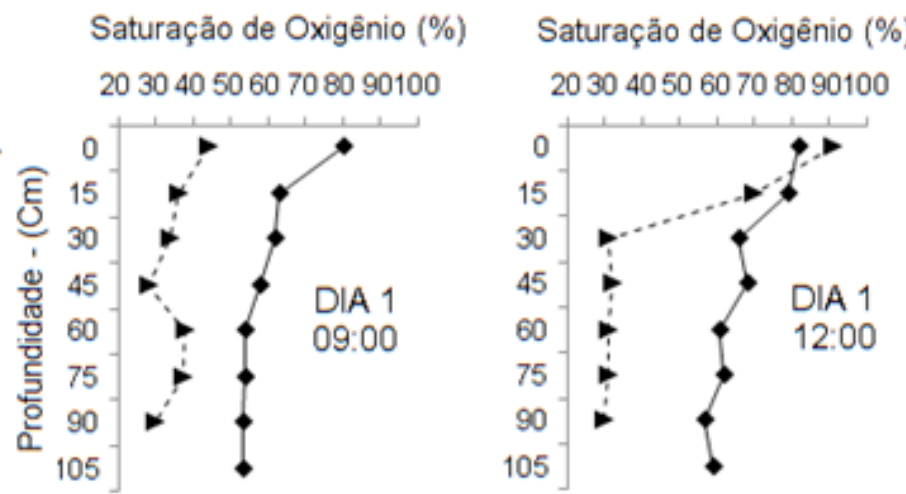

Saturação de Oxigênio (\%)

2030405060708090100

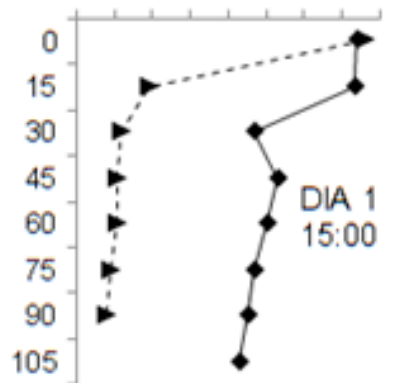



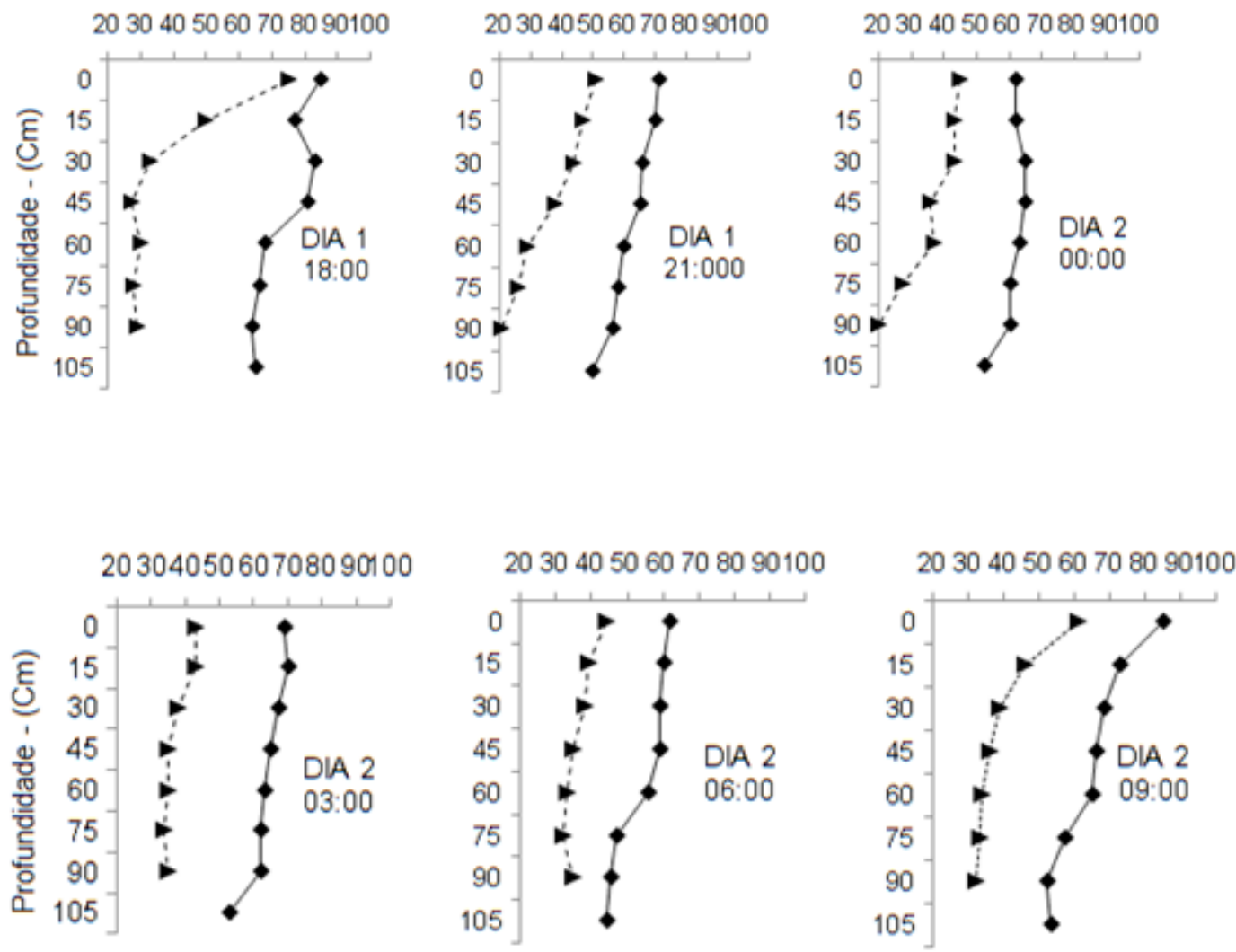

$\stackrel{--}{*}=$ Viveiro Escavado; $\rightarrow-=$ Viveiro de Barragem

Ferraz e Amaral (2011), analisando viveiros de cultivo de tilápias, encontraram porcentagem de saturação do oxigênio dissolvido que chegou a $116,27 \%$, no horário das $18 \mathrm{~h}$, evidenciando elevada atividade fotossintética. A partir desse horário, ocorreu uma redução gradativa na porcentagem de saturação, evidenciando-se uma relação direta com os processos biológicos de fotossíntese e respiração. Durante este estudo, foi possível observar essa queda na porcentagem de saturação de oxigênio, sendo mais acentuada no viveiro escavado. Já no viveiro de barragem permaneceu mais estável na coluna d'água (Gráfico 2). A variação pode estar correlacionada a de um maior aporte de matéria orgânica no viveiro escavado, associado à adubação e ao fornecimento de rações.

Durante o decorrer do experimento, a porcentagem de saturação de oxigênio foi superior no viveiro de barragem (Gráfico 2), o que nos permite determinar que esse ambiente, se comparado ao viveiro escavado, pode ser mais favorável para o cultivo de peixes, levando em consideração estes fatores.

De acordo com Alvarez (1999), nos viveiros de piscicultura que são cultivados em regime semi-intensivo, os processos internos de consumo e de produção de oxigênio pela comunidade fitoplanctônica, normalmente, são mais importantes do que os processos de difusão desse gás.

Segundo Boyd (1990), valores de oxigênio dissolvido (OD) acima de $4 \mathrm{mg} \cdot \mathrm{L}^{-1}$ são adequados para o cultivo de peixes. Sendo assim, o viveiro de barragem apresentou concentrações propícias para o cultivo de peixes, durante todo o período nictemeral ( 24 horas) e em toda coluna d'água. Este tipo de viveiro, permite o cultivo de espécies que habitem todos os ambientes, desde peixes pelágicos (que habitam no estrato do meio ou metalímnio), até peixes bentônicos (que habitam o fundo ou hipolímnio) (Gráfico 3). 
As variações nos níveis de OD, no viveiro escavado, foram mais acentuadas, o que poderia levar à morte de peixes. Também apresentou grandes diferenças entre a superfície e o fundo, tornando o fundo um local desfavorável para habitação de peixes que vivam nessa região do viveiro (Gráfico 3).

O manejo do OD é dispendioso, sendo realizado com adubações para aumentar o nível de fitoplâncton, ou com a utilização de aeradores. Diante disso, o cultivo em viveiros de barragem pode ser uma alternativa bem interessante para pequenos produtores, que não têm condições de fazer grandes investimentos. Segundo Kubitza (2003), o OD é essencial à vida dos organismos aquáticos e, quando em baixa concentração, pode retardar o crescimento, diminuir a eficiência alimentar e aumentar a incidência de doenças e de mortes.

\section{GRÁFICO 3 - Variação diária (Padrão nictemeral) do perfil vertical do Oxigênio Dissolvido.}
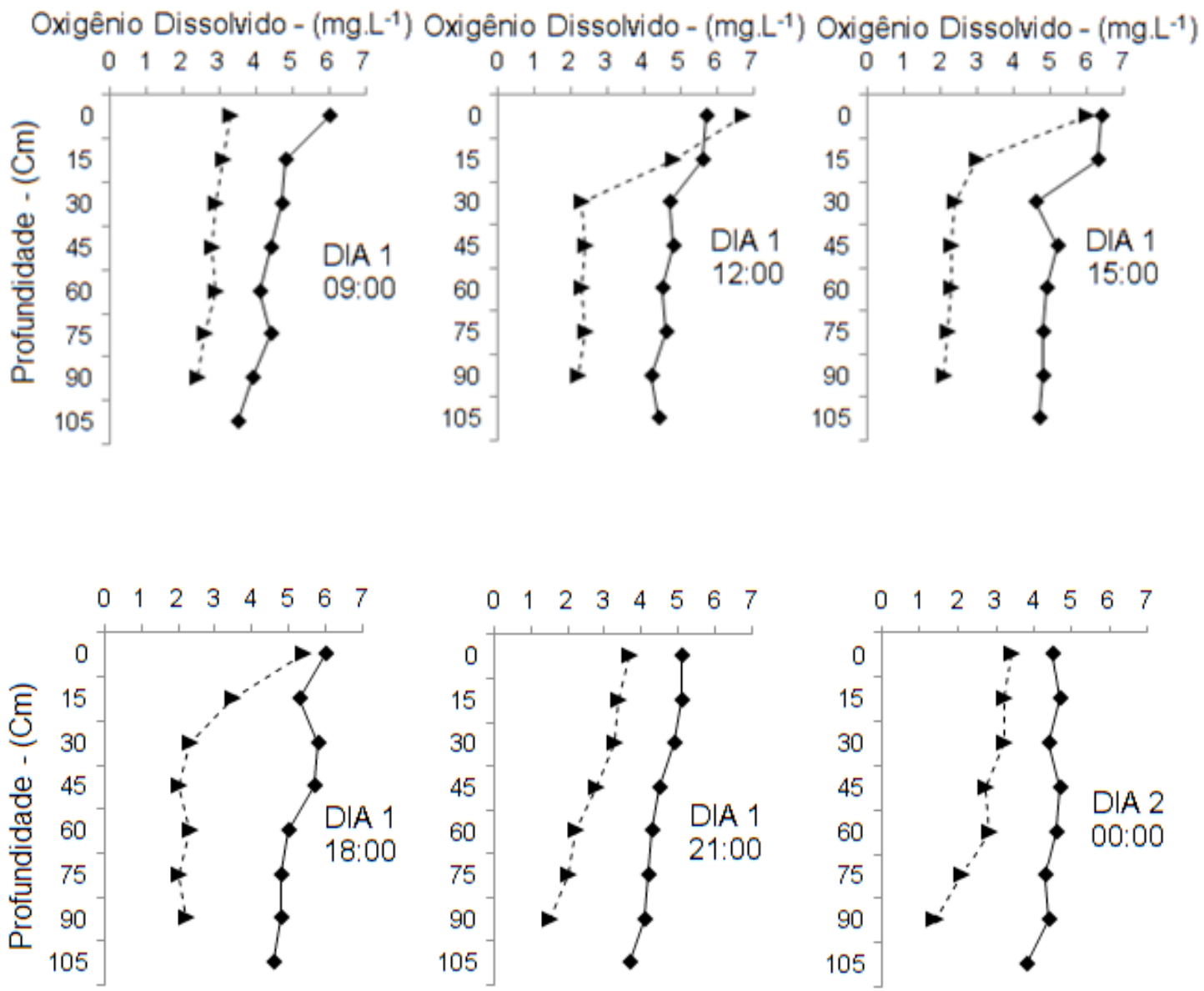

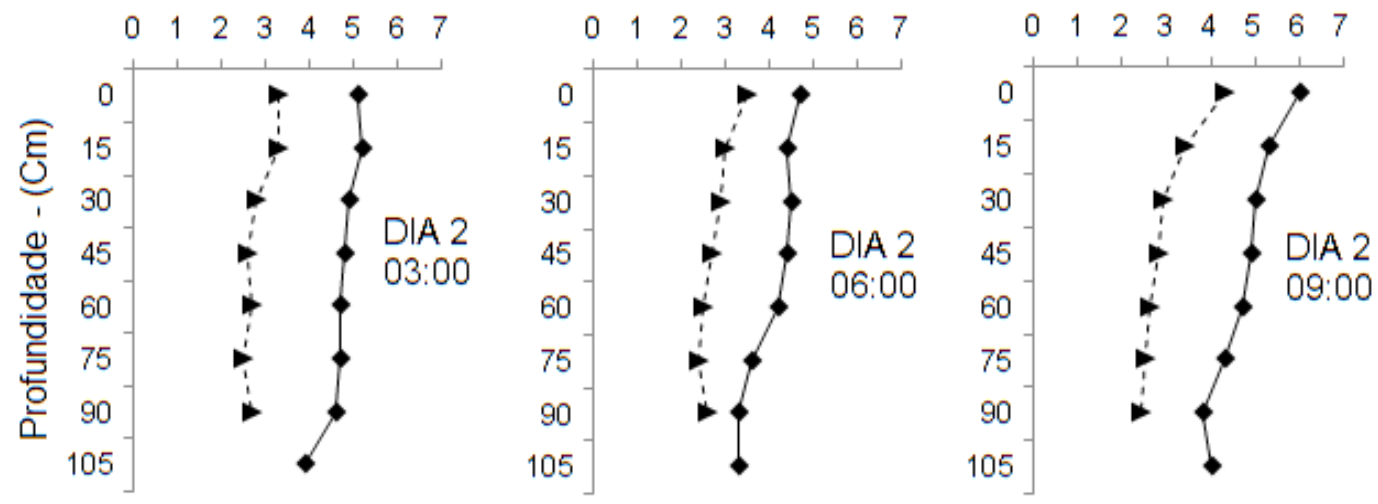

-- $\mathbf{A}^{--}=$Viveiro Escavado; $\longrightarrow-\mathbf{C}=$ Viveiro de Barragem.

O menor valor registrado de OD na coluna d'água foi no viveiro escavado, na profundidade de $90 \mathrm{~cm}$, à $0 \mathrm{~h}$, chegando ao nível de $1,4 \mathrm{mg} \cdot \mathrm{L}^{-1}$ (quase considerado estado de anoxia). $\mathrm{Na}$ comparação com os dados de OD do viveiro de barragem, que apresentou o menor valor de OD, 3,3mg. $\mathrm{L}^{-1}$, constata-se que o viveiro de barragem apresentou melhores condições naturais de cultivo. Henry-Silva et al. (2010) observaram que há uma amplitude de variação muito grande em relação às concentrações de oxigênio dissolvido ao longo do dia, no cultivo de camarões amazônicos, onde foram registradas as menores concentrações às $6 \mathrm{~h}$ e os maiores valores entre às $15 \mathrm{~h}$ e $18 \mathrm{~h}$.

Os níveis de saturação de oxigênio e concentrações de OD permaneceram superiores no viveiro de barragem, de forma significativa em todo o perfil da coluna d'água (Tabela 1), assim como em todos os horários. Esta superioridade significativa pode estar relacionada com a baixa quantidade de ração aplicada no viveiro de barragem, pela variação de espécies no local, e por habitarem estratos diferentes.

Tabela 1 - Análise estatística comparativa das variáveis, temperatura, saturação de oxigênio e concentração de oxigênio dissolvido, no perfil da coluna d'água.

\begin{tabular}{cccc}
\hline Hora & Temperatura $\left({ }^{\circ} \mathrm{C}\right)$ & Sat. Oxigênio (\%) & Oxig. Dissolvido (mg. $\left.\mathbf{L}^{-1}\right)$ \\
\hline $09: 00$ & $2,279^{*}$ & $6,169^{*}$ & $5,342^{*}$ \\
$12: 00$ & 0,953 & $2,29^{*}$ & $2,313^{*}$ \\
$15: 00$ & 1,033 & $3,36^{*}$ & $4,107^{*}$ \\
$18: 00$ & 1,297 & $4,904^{*}$ & $5,037^{*}$ \\
$21: 00$ & $3,659^{*}$ & $5,21^{*}$ & $5,149^{*}$ \\
$00: 00$ & $9,153^{*}$ & $6,837^{*}$ & $6,347^{*}$ \\
$03: 00$ & $19,117^{*}$ & $10,712^{*}$ & $10,018^{*}$ \\
$06: 00$ & $17,98^{*}$ & $5,509^{*}$ & $4,978^{*}$ \\
$09: 00$ & 0,263 & $4,458^{*}$ & $4,901^{*}$ \\
\hline
\end{tabular}

Valores de (t) a 5\% de significância. $P<0,05$.

* significativo, segundo teste (t) a $5 \%$ de significância. 


\section{Conclusão}

Este trabalho nos permitiu concluir que as variações nictemerais (24h) na coluna d'água foram mais abruptas e maiores no viveiro escavado. Portanto, os viveiros de barragem, são ambientes que podem trazer maior benefício, se considerarmos os fatores sociais e financeiros, pois favorecem melhores condições de cultivo.

\section{Referências}

ALVAREZ, E. J. A. Dinâmica de algumas variáveis limnológicas em tanques de larvicultura de Brycon orbignyanus sob dois tipos de tratamentos alimentares. Dissertação (Mestrado). Universidade Estadual Paulista “Julio de Mesquita Filho”UNESP, Jaboticabal-SP, 1999.

BARBOSA, F.A.R.; PADISÁK, J. The forgotten lake stratification pattern: atelomixis, and its ecological importance. Verhandlungen der Internationalen Vereinigung fur Theoretische und Angewandte Limnologie, vol. 28, p. 1385-1395, 2002.

BARRETO, L. E. G. S.; IGARASHI, M. A.; HAYASHI, C. Nictemeral variations for pacu Caranha, Piaractus mesopotamicus (Holmberg, 1887) culture in single - phase. Brazilian Archives Biology and Technology, vol. 53, n. 4, pp. 873-881, 2010.

BORGES, L. C.; FERREIRA, D. F. Comparação de duas aproximações do teste $t$ com variâncias heteregêneas através de simulação. Ciência e Agrotecnologia, v.23, n.2, p.390403, 1999.

BOYD, C. E. Water quality in ponds for aquaculture. Alabama: Birmingham Publishing, 1990.

BRITO, R. et al. Diel variation in the catch of the shrimp Farfantepenaeus duorarum (Decapoda, Penaeidae) and lenght-weight relationship, in a nursery area of the Terminos Lagoon Mexico. Revista de Biología Tropical, vol. 65, n. 1, pp. 65-75.

CAVALCANTE, L. M. Zoneamento geológico entre feijó e Mancio lima Cruzeiro do Sul- Acre. www.infoteca. Cnptia. Embrapa. Disponível online: Br/bitstrean/ doc/5059773/1/doc99. Pdf. Acessado em 30/05/2014. 2005.

CAVALCANTE, L. M. Relatório sobre a Geologia do Estado do Acre. Rio Branco: SEMA/IMAC. (texto integrante do eixo recursos natural do ZEE Fase II). 25 p 2006.

CYRINO. J.E.P.; SAMPAIO DE OLIVEIRA. A.M.B.M.; COSTA. A.B. Curso de Atualização em Piscicultura. Piracicaba, SP. Fundação de estudos agrários "Luiz de Queiroz", 1987.

DINIZ, C. R.; CEBALlOS, B. S. O.; PEDROSA, A. S.; KONIG, A.; BARBOSA, 
J. E. L. Distribuição vertical e dinâmica nictemeral de parâmetros físico-quimicos e biológicos do açude de Bodocongo - PB. XXVIII Congresso Interamericano de Engenharia Sanitária e Ambiental. México, 2002.

ESTEVES, F.A., BOZELLI, R. L., CAMARGO, A. F.M., ROLAND, F. \&THOMAZ, S.M. Variação diária (24h) de temperatura, oxigênio dissolvido, $\mathrm{pH}$ e alcalinidade em duas lagoas costeiras do Estado do Rio de Janeiro e suas implicações no metabolismo destes ecossistemas. Acta Limnologica Brasiliensia, v.2, p.99-127, 1988.

ESTEVES, F. A. Fundamentos de Limnologia. 2a ${ }^{a}$ ed. Rio de Janeiro: Interciência, 1998. FERNANDES, L. F.; WOSIAK,A. C.; DOMINGUES, L.; PACHECO, C. V.; LAGOS, P. E. Comunidades fitoplanctônicas em ambientes lênticos. Capítulo 10, p. 1- 39, 2004.

FERRAZ, D. R., AMARAL, A. A. Variação nictemeral dos parâmetros físico-químicos da água de um viveiro de cultivo de tilápias. XIV Encontro Latino Americano de Iniciação Científica, 2011.

FISHER, T. R.; MELACK, J. M.; ROBERTSON, B.; HARDY, E.R. Vertical distribution of zooplankton and physico-chemical conditions during a 24-hour period in an Amazon floodplain lake - Lago Calado, Brazil. Acta amazonica, v. 13, v. 3-4: p. 475-487, 1983.

HENRY-SILVA, G.G.; CAMARGO, A.F.M. Tratamento de efluentes por macrófitas aquáticas flutuantes. Revista Brasileira de Zootecnia, v.37, n.2, p.181-188, 2008.

HENRY-SILVA, G.G.; CAMARGO, A.F.M.; PONTES, C.S.; MIYASE, L. K. Características limnológicas da coluna d'água e dos efluentes de viveiros de criação de camarões-da-amazônia. Revista Brasileira de Zootecnia, v. 39, n. 10, p. 2099-2107, 2010.

KELLER T.; ASSAD E.D.; LIMA, P.R.S.R. Regiões pluviometricamente homogêneas o Brasil. Pesquisa Agropecuária Brasileira, vol. 40, n. 4, p. 311-322. 2005.

KUBITZA, F. Nutrição e alimentação dos peixes cultivados. 3ª. Ed., Jundiaí, Brasil, 123p., 1999.

KUBITZA, F.; ONO, E.A. Projetos Aqüícolas: Planejamento e Avaliação Econômica, $1^{\text {a }}$ ed. Jundiaí, 2004.

KUBITZA, F. Qualidade da água no cultivo de peixes e camarões. 3.ed.Jundiaí: ESALQ/ USP, 2003.

OSTRENSKY A.; BOEGER. W. Piscicultura: fundamentos e técnicas de manejo. Guaíba: Agropecuária, 1998. . 
PÁDUA, D. M. C. Fundamentos de piscicultura. 2a . ed. Goiânia: Ed. da UCG, 2001.

SILVEIRA, M. Vegetação e flora das campinaranas do sudoeste amazônico. SOS Amazônica: Relatório Técnico, Disponível online em: http://philip.inpa.gov.br/publ_ livres/Dossie/Mad/Outros\%20documentos/VegetacaoeFloraCampinaranas.pdf $28 \mathrm{p}$. Acesso em 30/05/2014. 2003.

STATSOFT. Inc. Statistica for Windows (data analysis software system), version 7.1. Statsoft, Tulsa, Oklahoma (USA), 2005.

TUNDISI, J. G.; MATSUMURA-TUNDISI, T. Plankton diversity in a warm monomictic lake (Dom Helvecio, Minas Gerais) and a Polymictic reservoir (Barra Bonita): A comparative analysis of the intermediate disturbance hypothesis. Anais da Academia Brasileira de Ciências, v. 66, n. 1, p. 15-28, 1994.

VINATEA ARANA, L. Princípios químicos de qualidade da água em aquicultura: uma revisão para peixes e camarões. $2^{\mathrm{a}}$. ed. Florianópolis: UFSC, 2004. 